\title{
Quantum energy gap in two quasi-one-dimensional $S=1$ Heisenberg antiferromagnets (invited)
}

\author{
J.P. Renard \\ Insitut d'Electronique Fondamentale, CNRS UA 22, Bât. 220, Université Paris-Sud, 91405 , \\ Orsay C'́dex, France \\ M. Verdaguer \\ Laboratoire S.E.T., CNRS UA 420, Bât. 420, Université Paris-Sud, 91405 , Orsay Cédex, France \\ L. P. Regnault, W. A. C. Erkelens, and a. Rossat-Mignod \\ C.E.N.G., DRF/SPh-MDN, 85X, 38041, Grenoble Cedex, France \\ J. Ribas \\ Departamento de Quimica, Universidad de Barcelona. Spain
}

W. G. Stirling and $C$. Vettier

Institut Laue Langevin, 156X, 38042, Grenobie Cédex, France

Following the Haldane conjecture, the antiferromagnetic (AF) Heisenberg chain of integer spins has a singlet ground state separated from the excited states by an energy gap. Recent numerical calculations on finite $\mathrm{AF}$ chains with $S=1$ supported this conjecture and provided an approximate value for the energy gap: $E_{G} \simeq 0.4|J|$, where $J$ is the intrachain exchange interaction. We report experimental studies on two $\mathrm{Ni}$ (II) quasi-one-dimensional (ID) AF with large intrachain interaction, $J / k \approx-50 \mathrm{~K}, \mathrm{Ni}\left(\mathrm{C}_{2} \mathrm{H}_{8} \mathrm{~N}_{2}\right)_{2} \mathrm{NO}_{2}\left(\mathrm{ClO}_{4}\right)(\mathrm{NENP})$ and $\mathrm{Ni}\left(\mathrm{C}_{3} \mathrm{H}_{10} \mathrm{~N}_{2}\right)_{2} \mathrm{NO}_{2}\left(\mathrm{ClO}_{4}\right)$, (NINO). In both compounds, the magnetic susceptibility along the three crystal axes steeply decreases below $T \simeq 15 \mathrm{~K}$ and no 30 long-range magnetic order could be detected down to $1.2 \mathrm{~K}$. These features are consistent with the Haldare conjecture. Inelastic neutron scattering experiments performed on NENP show two energy gaps, with an average value of about $0.4|J|$, which are explained by a splitting of the Haldane gap by singheion anisotropy.

\section{INTROOUCTION}

The interest in quantum antiferromagnetic chains has been strongly renewed by the recent theoretical results of

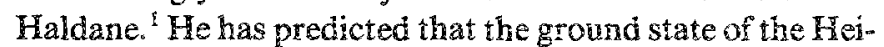
senberg chain, at $T=0 \mathrm{~K}$, is basically different for integer spin yalues than for half-integer spin values. In contrast to the $S=1 / 2,1 \mathrm{D}$ Heisenberg antiferromagnet (1D-FAF) which exhibits a continuum of excited states without gap from the ground state, ${ }^{2}$ the 1D-HAF with integer spin would exhibit a nonmagnetic singlet ground state separated from the excited states by an energy gap; an exponentiai decay of the spin correlations at $T=0 \mathrm{~K}$ is associated with the existence of the gap.

This result, which is rather surprising owing to the rotational invariance of the Heisenberg hamiltonian, is now well supported by numerical calculations on finite rings for $S=1 .^{3-6}$ For the $S=1,1 D-H A F$, a reliable estimation of the energy gap, $E_{G}=0.41|J|$, where $J$ is the nearest-neighbor exchange integral, has been obtained from Monte Carlo calculations up to 32 spins. ${ }^{7}$ The effect of magnetic anisotropy has also been studied on the following Hamiltonian:

\footnotetext{
"Present address: Kamerlingh Onnes Laboratorium, P.O. Box 9506,2300 RA Leiden, The Netherlands.
}

$$
\begin{aligned}
\mathscr{P}= & \sum_{i}\left[-J\left(S_{i}^{x} S_{i+1}^{x}+S_{i}^{y} S_{i+1}^{y}\right.\right. \\
& \left.\left.+\lambda S_{i}^{z} S_{i+1}^{z}\right)+D\left(S_{i}^{z}\right)^{2}\right]
\end{aligned}
$$

where $J$ is the intrachain exchange interaction, $1-\lambda$ the exchange anisotropy, and $D$ the single ion anisotropy.

It appears that the symmetry breaking terms do not destroy Haldane's gap if they are sufficiently small with respect to the isotropic coupling $J$.

The existence of such a gap would greatly affect the lowtemperature magnetic properties. As the temperature decreases below $E_{G} / k$, the magnetic susceptibility must decrease rapidly and tend to a value close to zero, independently of the magnetic field orientation. In addition, since the ID correlation length is severely limited as $T \rightarrow 0$, the interchain interaction $J^{\prime}$ which is always present in real quasi- $1 D$ magnetic systems would be unable to induce threedimensional long-range order (3D-LRO), below a critical value of the ratio $|J / J|{ }^{8}$ This situation is very different for the gapless 1D-HAF which achieves 3D-LRO at $T_{N H}$ $\approx 2 S^{2}\left(\left|J \times J^{\prime} / k\right|\right)^{i / 2}$. Furthermore, it must be pointed out than an anisotropy gap induced by an additional Ising-like term would increase the correlation length for spin components along the easy axis and induce 3D-LRO at a temperacure still higher than $T_{N H}$. 
Owing to its drastic effect on the low-temperature magnetic behavior, it is surprising that Haldane's gap was not experimentally observed for a long time. In fact, until recently, most experimental studies of quantum effects were performed on $S=1 / 2$ chain compounds, which were thought to mimic the extreme quantum case. Inelastic neutron scattering experiments on the $S=1$, quasi-1D-HAF $\mathrm{CsNiCl}_{3}$ have shown the existence of an energy gap which cannot be simply interpreted as due to magnetic anisotropy. ${ }^{9,10}$ However, $\mathrm{CsNiCl}_{3}$ is by far the best candidate for a clear exper:mental evidence of Haldane's gap since it shows 3D-LRO at $T_{N}=4.85 \mathrm{~K}$. This $\mathrm{LRO}$, which is not expected for the ideal system with a gap, restricts the useful temperature range for the gap observation to $k T \cong E_{G}$, where thermal broadening is important. In a preliminary work, ${ }^{1}$ we have shown that $\mathrm{Ni}\left(\mathrm{C}_{2} \mathrm{H}_{8} \mathrm{~N}_{2}\right) \mathrm{NO}_{2} \mathrm{ClO}_{4}$ (NENP) has the expected magnetic behavior for a $S=1$ antiferromagnetic chain with a quantum gap. We report here a more extensive study of the magnetic properties of NENP at low temperature and the first experimental results on a new $S=1$ antiferromagnetic chain compound, $\mathrm{Ni}\left(\mathrm{C}_{3} \mathrm{H}_{20} \mathrm{~N}_{2}\right)_{2} \mathrm{NO}_{2}\left(\mathrm{ClO}_{4}\right)$ ( $\left.\mathrm{NINO}\right)$.

\section{CAYSTAL STRUCTURE OF NENP AND NINO}

NENP and NINO present very similar crystallographic structures: both crystallize in the orthorhornbic system. NENP can be described either in the $P n{ }_{2}, a$ or in the Pnma space group, ${ }^{12}$ whereas NINO belongs to the $P b n \hat{2}_{1}$ space group. ${ }^{13}$

$$
\begin{array}{ll}
\text { For NENR/A: } & a=15.223(4) ; \quad b=10.300(3) ; \\
& c=8.295(2) ; \quad z=4 . \\
\text { For NINO/A口: } & a=15.384(3) ; \quad b=10.590(2) ; \\
& c=8.507(2) ; \quad z=4 .
\end{array}
$$

In both compounds, the structure consists of nickel (II) ions chains disposed along the $b$ crystal axis (Fig. 1). The chains lie remote from each other, well isolated by the perchlorate anions. The nickel (II) ions are covalently linked by bridging nitrite groups bonded on one side by the nitrogen atom and on the other side by one of the oxygen atoms. The local stereochemistry of nickel is a distorted octahedron; the basal plane is roughly perpendicular to the $b$ chain axis built with four nitrogen atoms from two diamine molecules: ethane diamine in NERB and 1,3 propane diamine in NINO. The Ni-N distances are around $2.08 \AA$ in NENP and range from 2.05 to $2.14 \AA$ in NINO. The octahedron axial positions along the $b$ chain axis are occupied by a nitrogen atom of a bridging nitrite group ( $N-N=2.16 \AA$ in both compounds) and by an oxygen atom of another nitrite bridging group. The main structural difference is indeed the Ni-O distance [2.183(4) A in NENP and 2.282(3) A in NINO]. It can be pointed out that the change from ethane diamine in NENP to propane diamine in NINO not only allows us to modify the local stereochemistry of nickel, and therefore its anisotropy properties, but also suppresses the disorder observed in NENP at two levels: the nitrite groups positions and the ethane diamine conformation. This allows us to describe the NENP structure in the Pnma space group, which has an inversion center at nickel, whereas the nickel chains existing in the crystal and described in Ref. 12, like the one in NINO, do not really have a nuclear inversion center. The octahedral configuration ensures a kigh-spin state $S=1$ for the nickel (II) ions, giving rise to two magnetic orbitals per nickel (II) ion, where one is of $d_{x y}$ symmetry in the basal plane of the octahedron and the other is of $d z^{2}$ symmetry along the chain axis.

The nitrite groups allow a strong overlap of $d z^{2}$ magnetic orbitals along the chain: a strong antiferromagnetic interaction between nickel (II) ions is therefore foreseen.

\section{KACROSCOPIC MACNETC PROPERTES}

\section{A. Magnetic suscoptibllity OR NEP and NINO}

The magnetic susceptibility of single crystals of NENP and NINO along the three or thogonal crystal axes $a, b$, and $c$ versus temperature below $100 \mathrm{~K}$ is shown in Figs. 2 and 3 . The same qualitative behavior is observed for both compounds. For the three orientations, the susceptibility reaches a rounded maximum at about $60 \mathrm{~K}$ and falls down abruptly
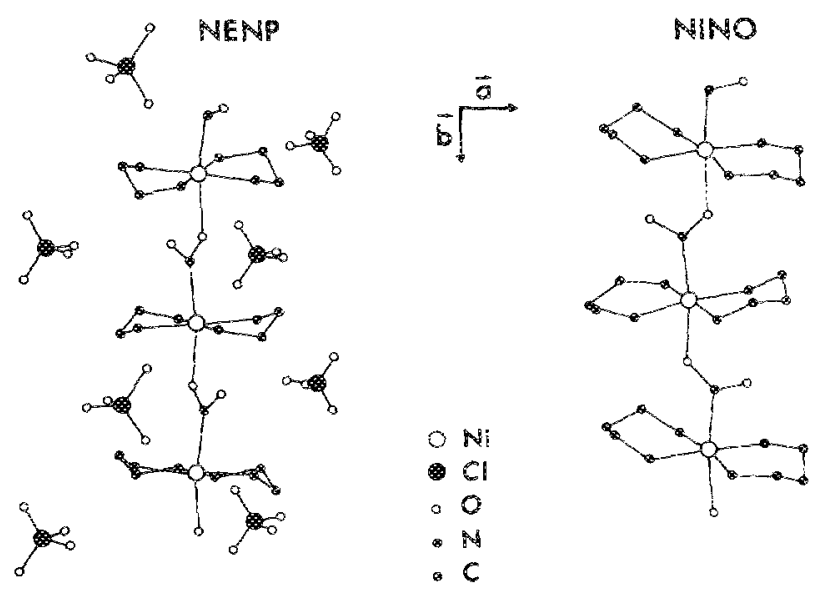

FIG. 1. Schematic view of the chain structure of $\mathrm{Ni}\left(\mathrm{C}_{2} \mathrm{H}_{8} \mathrm{~N}_{2}\right)_{2} \mathrm{NO}_{2}\left(\mathrm{ClO}_{4}\right)$ (NENP) and $\mathrm{Ni}\left(\mathrm{C}_{3} \mathrm{H}_{10} \mathrm{RN}_{2}\right)_{2} \mathrm{NO}_{2}\left(\mathrm{ClO}_{4}\right)$ (NINO).

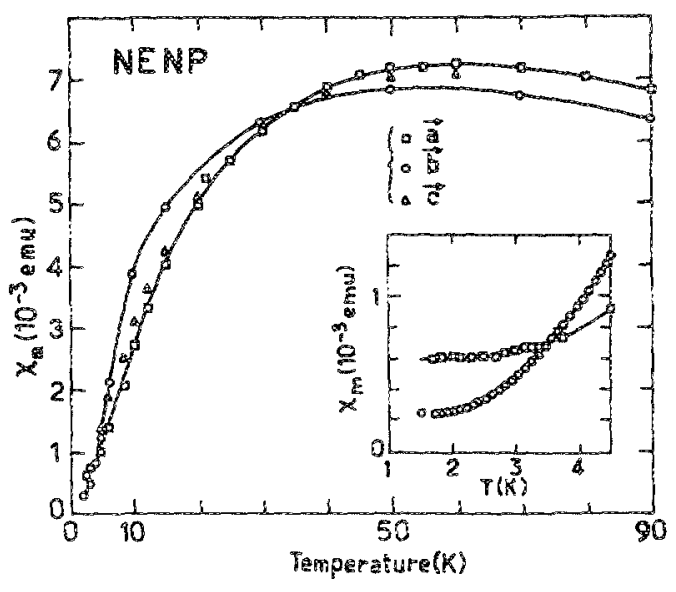

FIG. 2. Molar susceptibility of NENP corrected from diamagnetism along the three crystal axes $a(\square), b(O)$, and $c(\triangle)$ as a function of temperature. The solid lines are guides to the eye. 


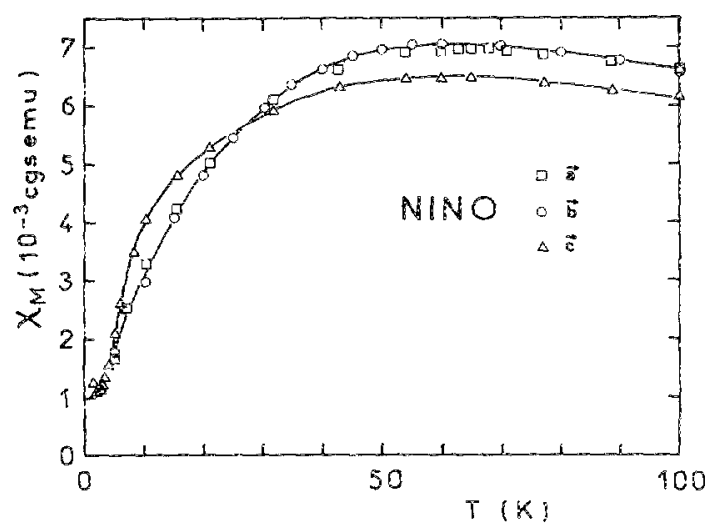

FIG. 3. Molar susceptibility of NINO, corrected from diamagnetism, along the three crystal axes $a(\square), b(O)$, and $c(\Delta)$ as a function of temperature The solid lines are guides to the eye

as the temperature is lowered below $15 \mathrm{~K}$. The limiting value $\chi(0)$ of the susceptibility (as $T$ tends to zero) is only a few percent of its maximum value $\chi_{\max }$. The low-terrperature behavior of the susceptibility is very different from the expected behavior of a gapless $1 D \cdot H A F$ for which $\chi(0) / \chi_{\max }$ is slightly smaller than $1\left[\chi(0) / \chi_{\max }=0.69\right.$ for $S=1 / 2$ and 0.83 for $S=\infty 1$. It is also different from the susceptibility behavior of an antiferromagnet with strong anisotropy for which the parallel susceptibility (If) falls to zero while the perpendicular (1) susceptibility is much smaller than $\chi_{\max }$ and nearly temperature independent. Furthermore, the susceptibility curves do not reveal any transition to a LRO antiferromagnetic phase. We conclude that the susceptibility of NENP and NINO is consistent with the existence of Haldane's gap. A rough estimate of $E_{G}$ is obtained for NENP by fitting the low-temperature experimental data $(T<5 \mathrm{~K})$ by the following relation:

$$
\chi(T)=\chi(0)+C \exp \left(-E_{g} / k T\right),
$$

with $E_{G} / k=11$ and $17 \mathrm{~K}$ for respective feld directions along $b$ axis and perpendicular to $b$. For NINO such an analysis was not done because the low-temperature data revealed an impurity contribution. However, the striking similarity between susceptibility temperature dependencies for NENP and NINO suggest gap values of the same order of magnitude. The detailed behavior of $\chi(T)$ as $T \rightarrow 0$ is clearly anisotropic as shown by Figs. 2 and 3. In the high-temperature range, Meyer et al. ${ }^{12}$ observed that along the three axes, the susceptibility data for NENP can be put in coincidence by simply introducing different $g$ factors: $g_{a}=2.23, g_{b}=2.15$, and $g_{c}=2.21$. The data are fitted by the high-temperature series expansion obtained by Weng ${ }^{14}$ for the Heisenberg hamiltonian with $\sharp=-33 \mathrm{~cm}^{-1}(-47.5 \mathrm{~K})$. In fact, a detailed analysis of our data near the broad susceptibility maximum using a least-squares fit reveals that the temperature of the maximum depends on the orientation (Fig. 4): For NENP, $T_{\max }=56 \mathrm{~K}$ for susceptibility along the $b$ axis and $T_{\max }=61 \mathrm{~K}$ perpendicular to $b$ axis. For NINO, $T_{\max }=66,65.4$, and $61.4 \mathrm{~K}$, respectively, for the $a, b$, and $c$ axes. Such large differences in the temperatures of the susceptibility maximum are not consistent with the small single ion anisotropy $D\left(S_{i}^{z}\right)^{2}$ with $D=1.5 \mathrm{~K}$ proposed for

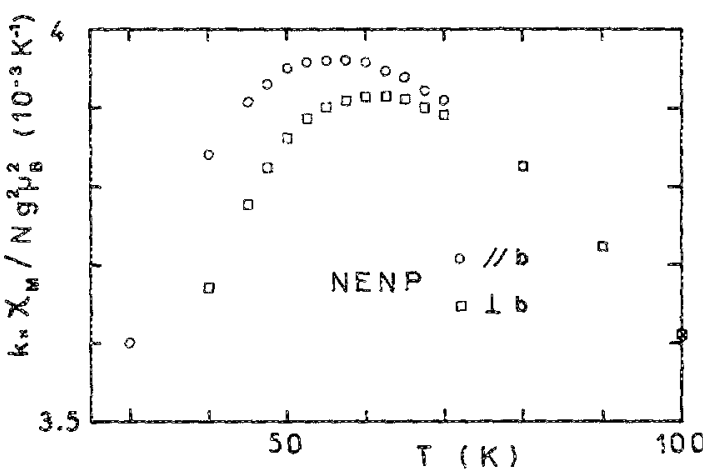

FIG. 4. Reduced molar susceptibility of NENP, $k \chi_{M} / N\left(g^{\alpha} \mu_{B}\right)^{2}$ for field orientation parallel $(O)$ and perpendicular $(\square)$ to the $b$ axis with $g^{\prime \prime}=2.15$ and $g^{+}=2.22$. Notice the different values for the temperature maximum.

NENP. ${ }^{12}$ In the absence of reliable calculations for the susceptibility of the anisotropic $S=1, \mathrm{AF}$, an evaluation of the single ion anisotropy of $\mathrm{Ni}^{2+}$, arising from a trigonal or tetragonal distortion of the ligand octahedra ${ }^{15}$ can be obtained by

$$
D=(\lambda / 2)\left(g^{\prime \prime}-g^{i}\right)
$$

where $\lambda$ is the spin-orbit coupling constant, $\lambda \simeq-250$ $\mathrm{cm}^{-1}$, for $\mathrm{Ni}^{2+}$, and $\mathrm{g}^{\prime \prime}$ and $\mathrm{g}^{1}$ the respective $g$ values parallel and perpendicular to the distortion axis $z$. For NENP, relation (3) gives $D \simeq 12 \mathrm{~K}$ and $z$ is along $b$, while for NINO, $D \simeq 18 \mathrm{~K}$ and $z$ is along $c$. These values of $D$ are very approximative since $\lambda$ and $g$ are not precisely known.

It should also be noticed that in spite of a larger $\mathrm{Ni}-\mathrm{Ni}$ distance, the $|J|$ value of NINO is close to that of NENP $\left(\simeq+8 \%\right.$ from average values of $T_{\max }$ and $\chi_{\max }$ ).

\section{B. High-fleld magnetizatlon of NENP}

The magnetic moment $M$ of a polycrystalline sample of NENP versus applied field up to 150 kOe at different ternperatures is shown in Fig. 5. At the highest temperature of the experiment, $T=50 \mathrm{~K}, M(H)$ is linear with a slope fairly

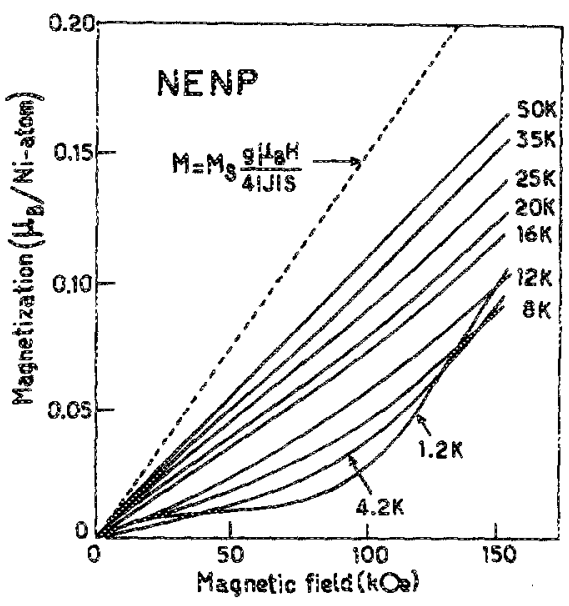

FIG. 5. Magnetization curves of NENP as a function of fieid and temperature. 
consistent with the average susceptibility measured in low field (see Sec. MI A). At the lowest temperature, $T=1.2 \mathrm{~K}$, the magnetic moment displays the interesting behavior predicted for the $S=1,1 D-H A F$ (Ref. 4): Neglecting a small parasitic contribution $\left(\approx 0.01 \mu_{B}\right.$ per Ni atom) related to impurities or finite chain units unavoidable in a powder, the magnetic moment remains very small up to $H \simeq 80 \mathrm{kOe}$ and then exhibits a linear increase at higher fields ( $H>100 \mathrm{kOe}$ ) with a slope close to the expected value for the $S=1,1 D$ HAF (Fig. 5). The critical field which breaks the energy gap is estimated to $H_{c}=90 \mathrm{kOe}$. The corresponding energy, $E_{G}=g \mu_{B} H_{c}$, is about $13 \mathrm{~K}$ for $g=2.2$, in good agreement with the average value $E_{G} \simeq 14 \mathrm{~K}_{2}$ obtained from low-temperature susceptibility.

\section{INELASTIC MEUTRON SCATEERIMG STUOY OF NENP}

The energy gap in the spectrum of magnetic excitations of NENP has been directly observed by inelastic neutron scattering (WNS). 1116 For the $S=1 / 2,1 \mathrm{D}-\mathrm{HAF}$, the spectrum of magnetic excitations in zero field consists of magnons with the well-known $1 D$ dispersion law ${ }^{2}$ :

$$
\hbar \omega_{q}=\pi|J| \times|\sin \pi q| \text {. }
$$

In the $S=1,1 D-H A F$ one expects, following Haldane's conjecture, the opening of a quantum gap $E_{G} \simeq 0.4|J|$ at $q=0$ and $q=1$. Our experiments revealed in zero field two energy gaps at $q=1$ associated with the magnetic fuctuations paralle ( $(\|)$ and perpendicular ( 1 ) to the chain axis $b$, at respective energies $E_{G}^{\|} \simeq 2.6 \mathrm{meV}(\simeq 30 \mathrm{~K})$ and $E_{\sigma}^{\perp} \simeq 1.2$ meV $(\simeq 14 \mathrm{~K})$. The dispersion curve of the two modes near $q=1$, shown in Fig. 6 , is well fitted by the following dispersion law:

$$
\left.\hbar \omega_{q}=\left[(E)_{G}^{\| \cdot 1}\right)^{2}+\left(2 \pi|J| S q^{*}\right)^{2}\right]^{1 / 2}
$$

with $q^{*}=1-q$ and $|J|=55 \mathrm{~K}$, in satisfactory agreement with the $J$ values deduced from susceptibility ${ }^{12}$ and specific heat measurements. ${ }^{17} \mathrm{~A}$ small wave-vector dependence ( $\approx 0.2 \mathrm{meV}$ ) of the lowest-energy mode is observed along the [100] direction, perpendicular to the chain axis (Fig. 6).

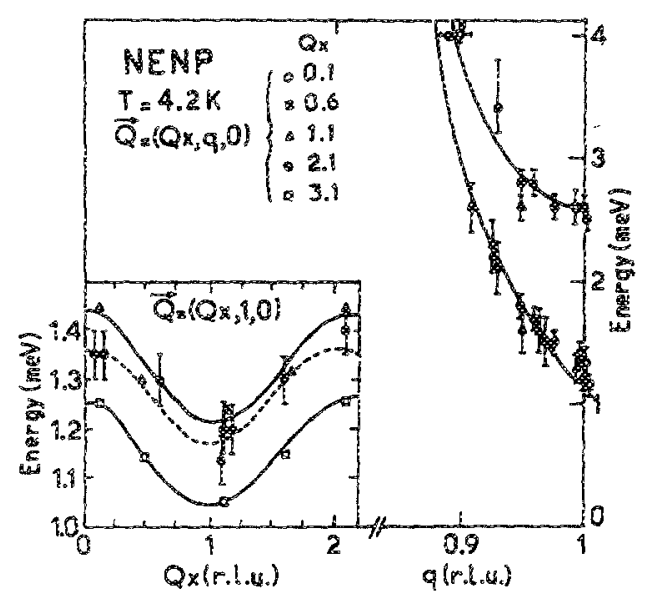

FIG. 6. Dispersion curves along the chain axis $b$ and perpendicular to the chain axis in NENP. The lines are fits to the spin-wave theory.
We attribute this perpendicular dispersion to the weak interchain interaction $J^{\prime}$. The experimental data can be fitted by a classical spin-wave approximation with $\left|J^{\prime} / J\right| \approx 4 \times 10^{-4}$. This low value of the ratio of interchain to intrachain interaction establishes the good 10 character of NENP.

The temperature dependence of the lowest-energy mode is shown in Fig. 7. The energy increases with increasing temperature (up to $E_{g}=0.35|J|$ ), at the highest temperature $(\approx 20 \mathrm{~K}$ ) where the excitation is observable. This energy increase is accompanied by a progressive damping of the excitation, which can be characterized by a damping parameter $\Gamma=\Gamma_{0} \exp \left(-E_{G} / k T\right)$ with $E_{G} \simeq 14 \mathrm{~K}$ and $\Gamma_{0} \simeq 20$ K.

The effect of a magnetic fied parallel to the $c$ crystal axis is shown in Fig. 8. The lowest-energy mode has an energy which is neariy independent of the field up to the critical field $H_{c} \approx 90 \mathrm{kOe}$ while its intensity decreases by a factor of 2 from $H=0$ to $H=H_{c}$. A stronger increase of the energy of the highest mode is observed. For field values around $H_{c}$, the situation becomes simlar to that observed in TMMC, $^{18}$ a quasiclassical $(S=5 / 2)$ 1D-AF with $X Y$ anisotropy, in which there is an anticrossing between the out-of-plane fluctuations and the two-magnon modes due to the canting of the spins induced by the high applied feld. ${ }^{19}$

Finally, it must be pointed out that both the temperature and low-field behavior of the lowest-energy excitation in NENP cannot be explained by the classical model of $1 D-A F$ with Ising anisotropy. For this model, a decrease of the energy gap with increasing temperature and a strong field dependence are expected.

\section{Y. DISGUSSION}

The susceptibility, magnetization, and INS experiments described in Secs. III and IV show the existence of energy gaps in the $S=1$ quasi-1D-AF NENP and NINO, in agreement with the theoretical predictions of $\mathrm{Haldane}^{1}$ The absence of any magnetic $\mathrm{LRO}$ at low temperature down to 1.2 $\mathrm{K}$ for $\mathrm{NENP}, 1.5 \mathrm{~K}$ for $\mathrm{NINO}$ in all experimental studies (susceptibility, specific keat, ${ }^{17}$ neutron scattering, and nuclear magnetic resonance ${ }^{20}$ ) is fully consistent with the severe limitation to a few spins of the $1 D$ correlation length as $T$ tends to zero. The magnetic anisotropy of NENY and

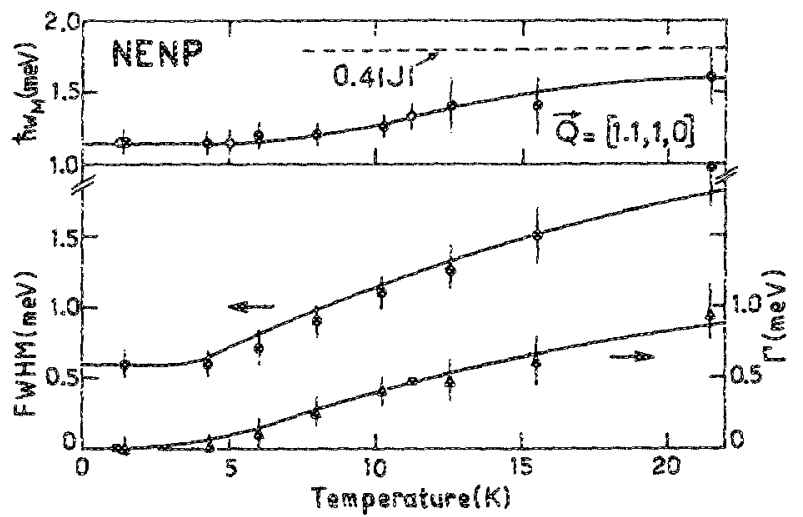

FIG. 7. Temperature dependencies of the peak position $\left(\omega_{\text {a }}\right)$, experimental width (FWHM), and damping parameter $(\Gamma)$ of the lowest-energy mode in NENP. 


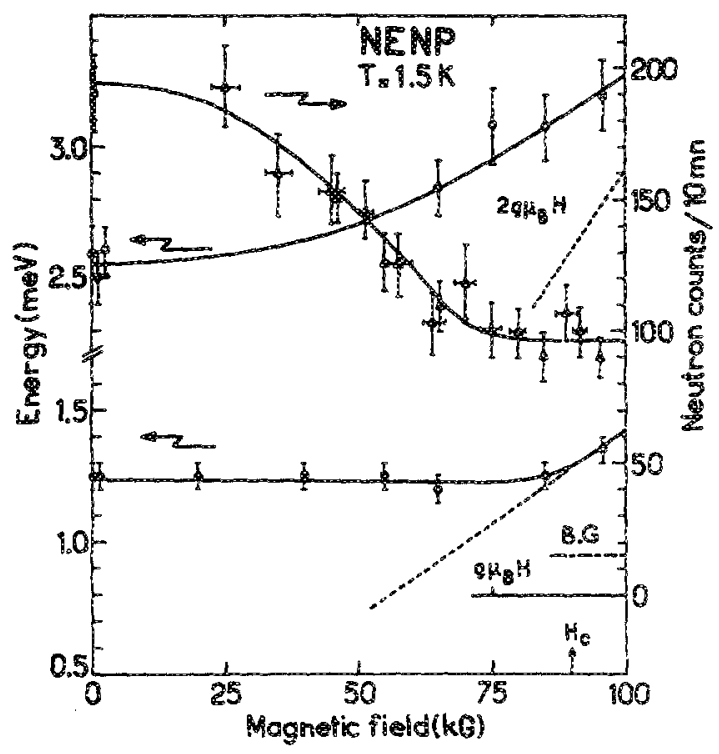

FIG. 8. Field dependencies of the magnetic excitations around $q=1$ : energies $(0,0)$ and intensity at maximum for $\mathrm{Q}=(0.5,1,0)$. The full lines are guides to the eye. The dashed lines represent the classical one- and twomagnon modes.

NINO is not negligible with respect to $J$ since $|D / J| \approx 0.25$. However, this anisotropy is not large enough to destroy Hal. dane's gap. It splits the singlet-triplet gap at $E_{G}=0.41|J|$ of the ideal $S=1,1 \mathrm{D}-\mathrm{HAF}$ into two energy gaps, the lowest one corresponding to fuctuations in the easy plane at $E_{G}^{t}$ $=(0.275 \pm 0.02)|J|$ and the highest one corresponding to the fuctuations along the hard axis at $E_{G}$ $=(0.59 \pm 0.04)|J|$ for NENP. The average value of these gaps $\left(E E_{G}+E_{G}^{1}\right) / 2=(0.43 \pm 0.03)|J|$ is very close to the expected value for the isotropic chain.

The numerical calculations of Botet $e t$ al. ${ }^{3}$ allow one to relate the gap splitting to $D$ for $|D / J| \leqslant 1$ by $E !_{G}-E$ $=1.6 \mathrm{D}$. For $D=12 \mathrm{~K}$, the leads to $E_{G}^{\|}-E_{G}^{1} \simeq 19 \mathrm{~K}$, in satisfactory agreement with the observed splitting of $16 \mathrm{~K}$. The effect of increasing temperature is to reduce the effective anisotropy and thus to bring the system closer to the isotropic case. This is fully consistent with the observed temperature of the lowest-energy gap $E_{G}^{\perp}$ in NENP.

In our previous papers, ${ }^{11,16}$ another explanation for the existence of a gap had been raised: the Ni ions could form an alternating AF chain with two different exchange integrals $J$ and $\alpha J$. This explanation would imply a high sensitivity of the exchange integral to the $\mathrm{Ni}-\mathrm{Ni}$ distance, since the $\mathrm{Ni}$ chains appear to be uniform from the crystal structure determination. In fact, with the stereochemistry shown in Fig. $1, J$ is fairly insensitive to the Ni-Ni distance since NENP and NINO have about the same $J$ value with Ni-Ni distances which differ by about $0.1 \AA$. The possibility of exchange alternation therefore appears unlikely. Finally, the interest in finding new $S=1$ quasi-1D-AF with different values of exchange and anisotropy must be emphasized. This would allow us to confirm the relation between the energy gap and these parameters. The $S=1$ chain compounds $\mathrm{AgVP}_{2} \mathrm{~S}_{6}$ $(J \approx-450 \mathrm{~K})$ recently studied by Colombet et $a l^{21}$ and $\mathrm{Ni}\left(\mathrm{C}_{3} \mathrm{H}_{10} \mathrm{~N}_{2}\right)_{2} \mathrm{~N}_{3}\left(\mathrm{ClO}_{4}\right)(J \simeq-100 \mathrm{~K})^{\mathrm{i} 3}$ with a chain structure close to NENP and NINO seem to be promising owing to the marked decrease of their powder susceptibility. In another direction, theoretical calculations on the effect of a magnetic field on Haldane's gap are clearly needed.

\section{ACKNOWLEDGHENTS}

We wish to thank Dr. R. Jullien and Dr. H. J. Schulz for illuminating discussions, Dr. A. V. Chubukov for a reprint of his original paper in Soviet Physics JETP, Dr. P. Beauvillain for help in the treatment of susceptibility data, R. Vicente and M. Montfort for help in synthetic work, X. Solans for crystal structure determination of NINO, and Mrs. F. Genet for typing the manuscript.

19. D. M. Haldane, Phys. Lett. A 93, 464 (1983); Phys. Rev. Lett. 50, 1153 (1983).

2J. des Cloiseaux and J. J. Pearson, Phys. Rev. 128, 2131 (1962).

${ }^{3}$ R. Botet and R. Jullien, Phys. Rev, B 27, 613 (1983); R. Botet, R. Jullien, and M. Kolb, Phys. Rev, B 29, 5216 (1983).

${ }^{4} J$. B. Parkinson and J. C. Bonner, Phys. Rev. B 32, 4703 (1985).

${ }^{5}$ A. Moreo, Phys. Rev. B 35, 8562 (1987).

${ }^{6}$ H. Betsuyaku, Phys. Rev. B 36, 799 (1987).

${ }^{7}$ Vi. P. Nightingale and H. W. J. Blöte, Phys. Rev. B 33, 659 (1986).

'Yu. A. Kosevich and A. V. Chubukov, Soc. Phys. JETP 64, 654 (1986).

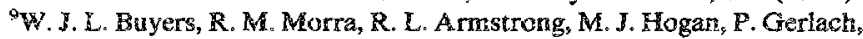
and K. Hirakawa, Phys. Rev. Lett. 56, 371 (1986).

${ }^{10}$ M. Steiner, K. Kakurai, J. K. Kjems, D. Petitgrand, and R. Pynn, J. Appl. Phys. 61, 3953 (1987).

'J. P. Renard, M. Verdaguer, L. P. Regnault, W. A. C. Erkelens, J. RossatMignod, and W. G. Stirling, Europhys. Lett. 3, 945 (1987).

${ }^{12}$ A. Meyer, A. Gleizes, J. J. Girerd, M. Verdaguer, and O. Kahn, Inorg. Chem. 21, 1729 (1982).

"M. Monfort, R. Vicente, J. Ribas, X. Solans, J. P. Renard, and M. Verdaguer, Inorg. Chem. (to be published).

${ }^{14} \mathrm{C}$. Y. Weng, Ph.D. Thesis, Camegie Institute of Technology (1968),

${ }^{15} \mathrm{~A}$. A bragam and $\mathrm{B}$. Bleaney, EPR of Transition Ions (Clarendon, Oxford, 1970), p. 450 .

I6L. P. Regnault, W. A. C. Erkelens, J. Rossat-Mignod, J. P. Renard, M. Yerdaguer, W. G. Stirling, and C. Vettier, Workshop on Magnetic Excitations and Fluctuations II, Torino, Villa Gualino, May 25-29, 1987 (Springer, Berlin) (to be published).

${ }^{27}$ J. Ferré and J. P. Jamet (to be published); W. A. C. Erkelens, 9. R. Nugteren, and $l . J$. de Jongh (privâte communication).

${ }^{18}$ I. U. Heilmann, J. X. Kjems, Y. Endohs, G. F. Reiter, and G. Shirane, Phys. Rev. B 24, 3939 (1981).

${ }^{19} \mathrm{~K}$. Osano, H. Shibs, and Y. Endoh, Prog. Theor. Phys. 67, 995 (1982).

${ }^{20}$ Proton magnetic resonance spectra of a monocrystal of NENP down to $1.2 \mathrm{~K}$ show a very small paramagnetic shift. The hyperfine splitting of the lines expected for a 3D ordered antiferromagnetic phase is not observed. ${ }^{21}$ P. Colombet, S. Lee, G. Ouvrard, and R. Brec, J. Chem. Res. (S), 134 (1987); S. Lee, P. Colombet, G. Ouvrard, and R. Brec, Mater. Res. Bull. 21, $917(1986)$. 